\title{
An intron enhancer recognized by splicing factors activates polyadenylation
}

\author{
Hua Lou, ${ }^{1}$ Robert F. Gagel, ${ }^{1}$ and Susan M. Berget ${ }^{2,3}$ \\ ${ }^{1}$ Section of Endocrinology, Department of Medical Specialties, The University of Texas M.D. Anderson Cancer Center, \\ Houston, Texas 77030 USA; $^{2}$ Verna and Marrs McClean Deptartment of Biochemistry, Baylor College of Medicine, \\ Houston, Texas 77030 USA
}

\begin{abstract}
Alternative processing of the pre-messenger RNA encoding calcitonin/calcitonin gene-related peptide (CT/CGRP) involves alternative inclusion of a 3'-terminal exon (exon 4) embedded within a six exon primary transcript. Expression of CT/CGRP in transgenic mice indicates that inclusion of exon 4 occurs in a wide variety of tissues, suggesting that the factors responsible for exon 4 inclusion are widely distributed. Inclusion of exon 4 requires an enhancer sequence located within the intron downstream of the poly(A) site of exon 4 . Here we show that the intron enhancer activated in vitro polyadenylation cleavage of precursor RNAs containing the CT/CGRP exon 4 poly(A) site or heterologous poly(A) sites. To our knowledge this is the first example of an intron-located enhancer that facilitates polyadenylation. Within the enhancer sequence is a $5^{\prime}$ splice site sequence immediately preceded by a pyrimidine tract. This $5^{\prime}$ splice site sequence was required for enhanced polyadenylation and was recognized by both U1 small nuclear ribonucleoproteins (snRNPs) and alternative splicing factor/splicing factor 2 (ASF/SF2). Enhancement of polyadenylation required U1 RNA, suggesting that the $5^{\prime}$ splice site sequence within the enhancer mediates enhancement via interaction with factors normally associated with functional $5^{\prime}$ splice sites. Mutation of the polypyrimidine track of the enhancer also inhibited in vitro polyadenylation cleavage. Oligonucleotide competitions and UV cross-linking indicated that the enhancer pyrimidine track binds the polypyrimidine tract binding protein (PTB), but not U2 snRNP auxiliary factor (U2AF), and that binding of PTB was required for maximal enhancer-mediated polyadenylation. These results suggest that the enhancer binds known splicing factors, and that binding of these factors activates polyadenylation cleavage. Furthermore, these results suggest that regulation of alternative processing of CT/CGRP could occur at the level of polyadenylation, rather than splicing.
\end{abstract}

[Key Words: Polyadenylation; splicing; polypyrimidine tract binding protein; calcitonin; intron enhancer]

Received October 6, 1995; revised version accepted November 21, 1995.

A relatively common pattern of differential RNA processing involves the alternative recognition of a $3^{\prime}$-terminal exon. The pre-messenger RNA coding for the peptides calcitonin $(\mathrm{CT})$ and calcitonin gene-related peptide (CGRP) undergoes such alternative processing (Amara et al. 1982). In this case, the fourth exon within the 6-exon primary transcript becomes an alternative 3 '-terminal exon (Fig. 1). Processing to produce an mRNA competent for production of calcitonin involves usage of exon 4 as a 3 '-terminal exon with concomitant polyadenylation at the end of exon 4. Processing to produce a mRNA competent for production of CGRP involves the exclusion of exon 4 and direct ligation of exon 3 to exon 5 , with polyadenylation occurring at the end of exon 6 . Introduction of the CT/CGRP gene into transgenic mice suggests that inclusion and polyadenylation of exon 4 and production of calcitonin occurs in a variety of tissues. In contrast, the skipping of exon 4 is restricted to only a few cell types including neuronal cells (Crenshaw et al. 1987).

${ }^{3}$ Corresponding author.
Like many differential exons, human CT/CGRP exon 4 can be characterized as having weak processing signals. The $3^{\prime}$ splice site of exon 4 is exceptionally weak. It contains a noncanonical branchpoint uridine (Adema et al. 1988). Mutation of this poor branchpoint to the consensus branchpoint adenosine causes constitutive inclusion of exon 4, even in cell types that never recognize the normal exon (Adema et al. 1990; Cote et al. 1991). The exon 4 poly(A) site can also be classified as weak when compared with other poly(A) sites, by the absence of a strong downstream GU- or U-rich sequence /Wahle 1995) and by low activity in in vitro polyadenylation assays (van Oers et al. 1994; see below). Weak differential exons are frequently associated with special enhancer sequences that facilitate exon recognition in the presence of accessory factors that bind to the enhancer (Hodges and Bernstein 1994). Several studies have suggested that sequences in and around CT/CGRP exon 4 in addition to the splice sites are necessary for exon 4 inclusion and might function as such enhancers (Cote et al. 1992; van Oers et al. 1994a; Lou et al. 1995). One of 
these elements is a sequence located 168 nucleotides downstream of the poly(A) site of exon 4 (Lou et al. 1995). Mutation or deletion of this intron element reduces exon 4 inclusion in vivo (Lou et al. 1995). Inside the intron element is a pseudo-5' splice site. Mutation of this $5^{\prime}$ splice site reduces exon 4 recognition in the natural CT/CGRP gene in vivo, suggesting that factors recognizing splice sites could participate in enhancer function.

Recognition of exon 4 could be influenced by factors that stimulate either splicing or polyadenylation. Although several intron-located enhancers that stimulate splicing have been discovered (Black 1992; Horabin and Schedl 1993; Huh and Hynes 1993; 1994), no intron element facilitating polyadenylation has been described. Furthermore, no intron enhancer sequence has been observed to contain a splice site, although a negative regulator of splicing containing a $5^{\prime}$ splice site has been described (Siebel et al. 1992). Factors recognizing 5' splice sites have been reported to regulate splice site choice via binding to $5^{\prime}$ splice sites (Siebel et al. 1994; Cáceres et al. 1994; Ge and Manley 1990; Krainer et al. 1990; Mayeda and Krainer 1992). U1 small nuclear ribonuclic-proteins (snRNPs) also bind to $5^{\prime}$ splice sites (Zhuang and Weiner 1986). Although snRNPs themselves have never been shown to regulate alternative processing, a constituent of U1 snRNPs, the A polypeptide has been shown to influence polyadenylation of poly(A) sites containing binding sites for the protein (Boelens et al. 1993; Lutz and Alwine 1994). Therefore, the CT/ CGRP enhancer has the potential to influence either splicing or polyadenylation via the binding of factors to the $5^{\prime}$ splice site within the enhancer.

Here we show that the CT/CGRP intron enhancer ac- tivates in vitro polyadenylation cleavage and binding of the 64-kD subunit of cleavage stimulation factor (CstF) to RNAs containing the CT/CGRP exon 4 poly(A) site, suggesting that at least one role of the enhancer in exon 4 recognition is via activation of polyadenylation of the exon 4 poly(A) site. The enhancer also activated polyadenylation of precursor RNAs containing heterologous poly(A) sites, indicating that the proteins binding to the enhancer can interact with the basal polyadenylation machinery. Activation was dependent on the presence of a wild-type 5' splice site sequence within the enhancer and U1 snRNA, supporting a role for U1 snRNPs in polyadenylation. The intron enhancer also contains a polypyrimidine tract and CAG immediately upstream of the pseudo $5^{\prime}$ splice site. Mutation of the polypyrimidine tract depressed polyadenylation cleavage and UV crosslinking of polypyrimidine tract binding protein (PTB) to the enhancer. An oligonucleotide representing the preferred polypyrimidine binding sites for PTB effectively competed polyadenylation cleavage, whereas an oligonucleotide specific for U2 snRNP auxiliay factor (U2AF) did not. Taken together these results suggest that the CT/ CGRP intron enhancer is recognized by splicing factors that normally recognize both $3^{\prime}$ and $5^{\prime}$ splice sites, and that both U1 snRNPs and PTB facilitate polyadenylation at the exon 4 poly(A) site.

\section{Results}

In vivo recognition of exon 4 requires a pseudo-5' splice site within the downstream intron

We have previously determined that the CT/CGRP gene contains an intron enhancer located 168 nucleotides downstream of the exon 4 poly(A) site (Fig. 1A). Deletion
A

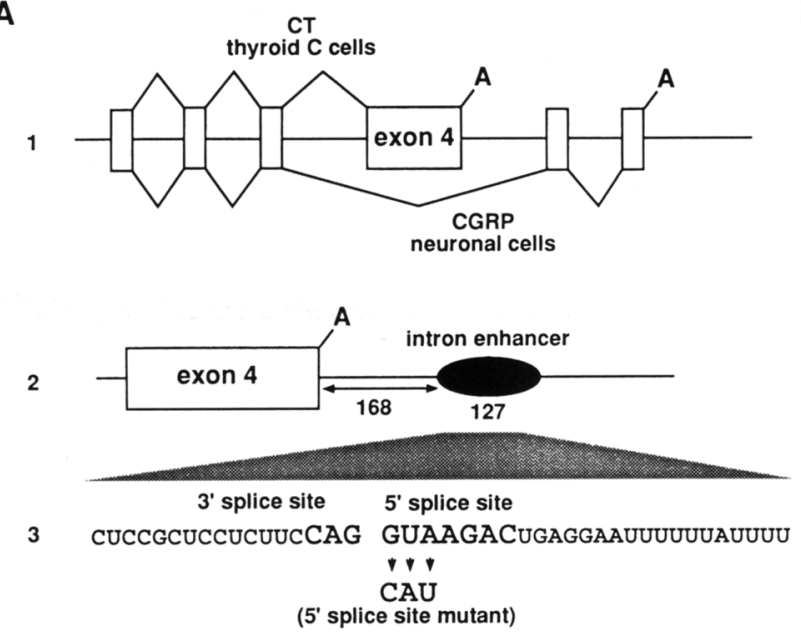

B

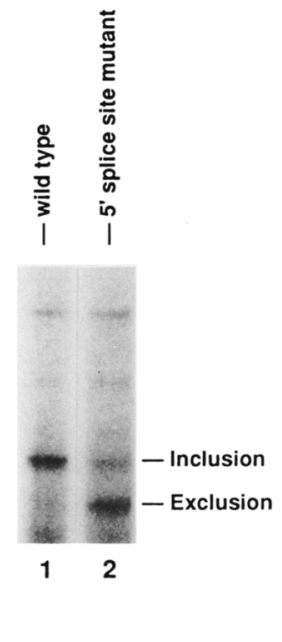

Figure 1. In vivo recognition of exon 4 requires a pseudo-5' splice site within the downstream intron. (A) A schematic diagram of the CT/CGRP gene and its resident intron enhancer. (Line 1) Alternative processing pattern of the CT/CGRP premRNA in thyroid and neuronal cells. (Line 2) Location of the intron enhancer downstream of exon 4 . The enhancer (127 nucleotides) is located 168 nucleotides downstream of the exon 4 poly(A) site and 346 nucleotides upstream of exon 5 . Throughout this report the intron enhancer is represented pictorially by a black oval. (Line 3) Sequence of the core sequence of the enhancer as revealed by previous in vivo experiments (Lou et al. 1995). The $5^{\prime}$ splice site sequence within the enhancer is shown in large letters. The $5^{\prime}$ splice site mutant used for most of the experiments in this study is indicated. $(B)$ In vivo inclusion of exon 4 is reduced by a mutation of the pseudo-5' splice site of the CT/CGRP enhancer. RT-PCR amplification of total cell RNA following transfection of HeLa cells with the wild-type human CT/CGRP gene (lane 1) or the human CT/CGRP gene containing a triple point mutation /altering the GUAAG pseudo-5' splice site of the intron enhancer to CAUAG; (lane 2). Product RNAs resulting from usage of exon 4 (inclusion) or skipping of exon 4 (exclusion) are indicated. Amplification used 2 oligonucleotides, one within exon 4 and one within exon 5, to permit simultaneous visualization of products that polyadenylated within exon 4 or skipped exon 4 , respectively, as described previously (Lou et al. 1995). 
of the enhancer or mutations within the enhancer depress recognition of exon 4 in vivo, resulting in production of a product RNA that contains exon 3 spliced directly to exon 5 (Lou et al. 1995). These results suggest that the enhancer operates by activating recognition of either the 3' splice site or the poly(A) site of exon 4 . The enhancer contains a sequence within it that resembles a 5 ' splice site. Point mutation of this sequence within the natural CT/CGRP gene so as to inactivate the sequence for use as a $5^{\prime}$ splice site depressed exon 4 recognition in vivo (Fig. 1B). These results suggested that the enhancer bound U1 snRNPs that in turn activated either splicing or polyadenylation of exon 4 .

\section{In vitro polyadenylation cleavage is stimulated by the intron enhancer}

To test whether the intron enhancer affected polyadenylation, in vitro polyadenylation assays were performed using in vitro precursor RNAs containing the CT/CGRP exon 4 poly(A) site with a wild-type or mutant intron enhancer (Fig. 2A). For these experiments a short precursor RNA was constructed that contained the last 244 of the 495 nucleotides of exon 4 and the first 257 nucleotides of intron 4 . This precursor RNA contained no functional splice sites for CT/CGRP splicing but did contain all known sequence elements for polyadenylation at exon 4 as well as the entire intron enhancer as determined by previous in vivo experiments (Lou et al. 1995). A precursor RNA containing the wild-type exon 4 poly(A) site with the enhancer was active for polyadenylation cleavage in an in vitro assay (Fig. 2B, lanes 2-4). Authenticity of the observed polyadenylation cleavage product was determined by creation of a mutant substrate in which the polyadenylation consensus sequence AAUAAA was altered to AAGAAA; the mutant RNA exhibited no polyadenylation cleavage product (Fig. 2B, lanes 5-7).

Two mutant precursor RNAs containing mutations of the $5^{\prime}$ splice site sequence within the intron enhancer were created and tested for in vitro polyadenylation cleavage. One mutation contained three altered nucleotides (positions +1 to +3 of the $5^{\prime}$ splice site sequence) and one contained a single altered nucleotide (position +1 of the $5^{\prime}$ splice site sequence) (Fig. 2B, lanes 8-13). The level of polyadenylation cleavage observed with both mutations was $\sim 25 \%$ of that observed with the wild-type precursor RNA. These results suggested that the intron enhancer functions to stimulate polyadenylation cleavage. Mutations that inactivated exon recogni-
A
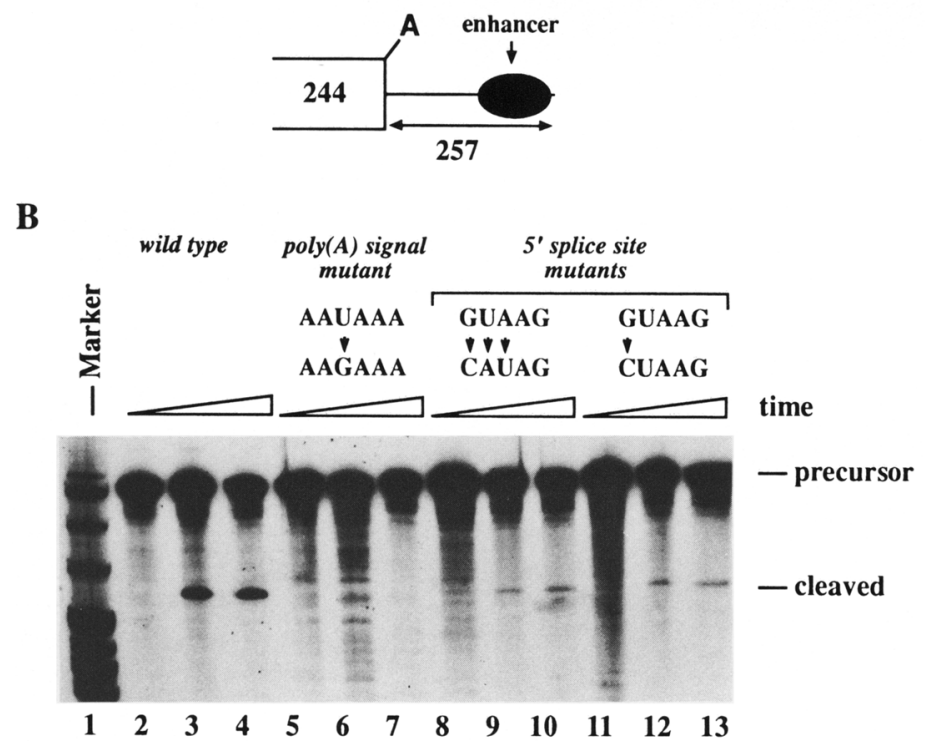

C

human core enhancer

CUCCGCUCCUCUUCCAG GUAAGACUgaggaAuUUUUUaUUUU ||||||||||||||||||||||$|\quad|||||||$ CUCUG*UCUUCCUUCAG GUAAGACUG*GGGUGCUUUCACUUU

mouse core enhancer

D

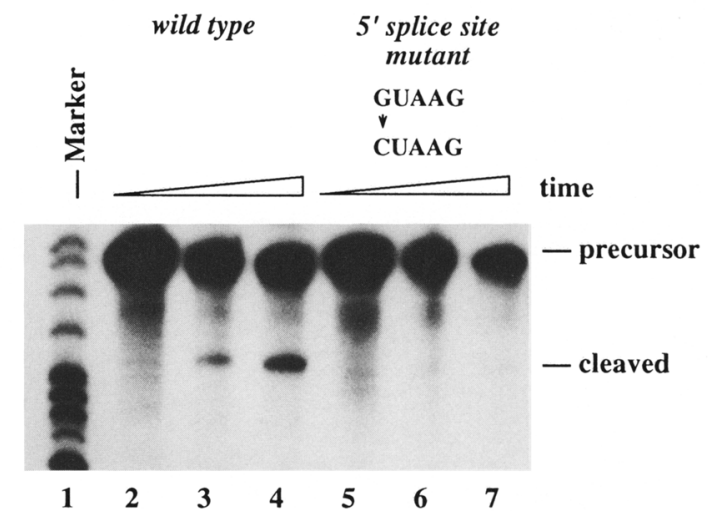

Figure 2. In vitro polyadenylation cleavage is stimulated by the intron enhancer. $(A)$ Schematic diagram of the wild-type CT/CGRP exon 4 precursor RNA used in this study. The utilized precursor RNA contained the last 244 nucleotides of exon 4 and the first 257 nucleotides of intron $4 .(B)$ In vitro polyadenylation cleavage assay of RNAs containing the human CT/CGRP exon 4 poly(A) site. In vitro polyadenylation cleavage assays were performed with the wild-type RNA containing the natural intron enhancer (lanes 2-4), a mutant in which the AAUAAA polyadenylation signal had been altered to AAGAAA (lanes 5-7), a triple point mutant in which the pseudo-5' splice site within the enhancer had been altered to CAUAG (lanes 8-10), and a single point mutant in which the pseudo-5' splice site within the enhancer had been altered to CUAAG (lanes 11-13). Precursor and cleaved product RNAs are indicated. Samples were taken for analysis at 0,20 , and $40 \mathrm{~min}$. $(C)$ Comparison of the human and mouse intron enhancer core sequences. $(D)$ In vitro polyadenylation cleavage assay of precursor RNA containing the mouse CT/CGRP exon 4 poly(A) site with a putative intron enhancer. A polyadenylation substrate from the mouse CT/CGRP gene similar to that constructed for the human gene was assayed. Both a wild-type version containing the putative intron enhancer region (lanes 2-4) and a mutant version altered for the pseudo-5' splice site in the mouse enhancer sequence (the mutation altered the 5' splice site from GUAAG to CUAAG; lanes 5-7) were assayed. 
tion in vivo (Lou et al. 1995) also inactivated polyadenylation cleavage in vitro. The observation that usage of exon 4 in vivo and polyadenylation cleavage at the exon 4 poly $(A)$ site in vitro were dependent on the same sequence suggested that the CT/CGRP intron enhancer functions in vivo to increase exon 4 recognition via an effect on polyadenylation cleavage.

The above experiments were performed with the human CT/CGRP gene. The mouse CT/CGRP gene undergoes a similar pattern of alternative splicing and polyadenylation (Benett and Amara 1993). The enhancer region of intron 4 of the CT/CGRP gene is highly conserved in human, rat, and mouse with a perfect fit between the enhancer-located 5 ' splice site sequences in these species (Fig. 2C; Lou et al. 1995). To test the generality of the observations shown in Figure 2 for the human gene, an in vitro precursor RNA for polyadenylation cleavage at exon 4 and containing the putative enhancer region was prepared from the mouse gene. A single point mutation at position +1 was introduced into the $5^{\prime}$ splice site portion of the mouse intron enhancer. As with the human gene, maximal in vitro polyadenylation cleavage of precursor RNAs containing the mouse exon 4 poly(A) site required a wild-type $5^{\prime}$ splice site sequence within the mouse enhancer (Fig. 2D).

\section{The CT/CGRP enhancer facilitates in vitro} polyadenylation of heterologous poly $(A)$ sites

From the previous observations we concluded that maximal polyadenylation cleavage at the exon 4 poly(A) site of CT/CGRP required the $5^{\prime}$ splice site sequence within the downstream enhancer. We then asked whether the CT/CGRP intron enhancer would activate in vitro polyadenylation of a heterologous poly(A) site. For this purpose a 127-nucleotide sequence from CT/CGRP intron
4, including the intron enhancer, was positioned $194 \mathrm{nu}-$ cleotides downstream of the poly(A) site of the Chinese hamster adenine phosphoribosyl transferase (APRT) gene (de Boer et al. 1989) and 124 nucleotides downstream of the poly(A) site of exon 6 of the human CT/ CGRP gene (Broad et al. 1989). Both a wild-type enhancer and an enhancer mutated for its constituent pseudo-5' splice site were added to the heterologous poly(A) sites. For this experiment precursors of equal length containing equal amounts of sequence downstream of the polyadenylation cleavage site, either with or without the intron enhancer, were employed.

Precursor RNAs containing both heterologous poly(A) sites were assayed for cleavage activity in the absence of the intron enhancer (Fig. 3, lanes 2-4, 11-13). In the absence of the enhancer, precursor RNA containing the APRT site was considerably more active than RNA containing the CT/CGRP exon 6 site. The intron enhancer stimulated the cleavage activity of both precursor RNAs containing heterologous poly(A) sites (Fig. 3, lanes 5-7, 14-16). Therefore, the CT/CGRP intron enhancer can activate polyadenylation cleavage of heterologous sites, regardless of the innate strength of the site. Activation was dependent on a wild-type $5^{\prime}$ splice site sequence within the enhancer (Fig. 3, lanes 8-10, 17-19), suggesting that the heterologous sites were being activated by the same mechanism as that operating to enhance CT/ CGRP exon 4 and that the factors binding to the enhancer can interact with the basal polyadenylation machinery.

\section{The intron enhancer stimulates binding of CstF} to the exon $4 \operatorname{poly}(A)$ site

One important component of the mammalian polyadenylation machinery is CstF (Takagaki et al. 1990). CstF

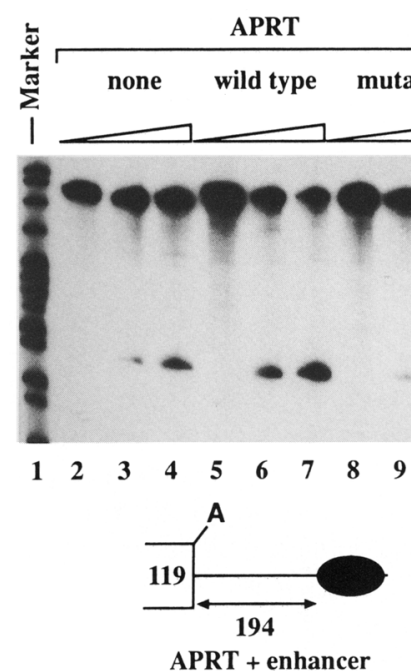

APRT + enhancer

\section{CT/CGRP exon 6}

none wild type mutant
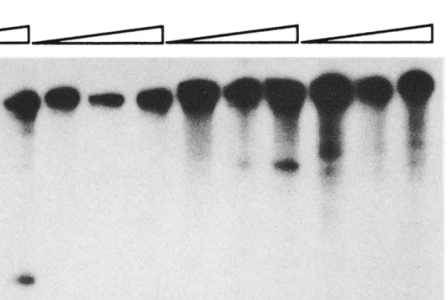

precursor RNA

enhancer

time

- precursor

- CT/CGRP exon 6 cleaved

- APRT

cleaved

enhancer (lanes 8-10 and 17-19, respectively). Samples were taken at 0,20, and $40 \mathrm{~min}$. The parental poly(A) sites for CT/CGRP exon 6 and APRT contained downstream sequences derived from pGEM vector of the same length as the CT/CGRP enhancer sequence added to the same parental poly(A) sites. 
is a heterotrimer whose binding to poly(A) sites can be monitored via UV cross-linking of its $64-\mathrm{kD}$ subunit to consensus sequences downstream of the poly(A) site (MacDonald et al. 1994). The cleavage activity of RNAs containing a poly $(\mathrm{A})$ site in vitro is proportional to the level of UV cross-linking to CstF observed for that site. Therefore, we predicted that the CT/CGRP intron enhancer might stimulate UV cross-linking of RNAs containing the exon 4 poly(A) site to CstF. We tested the ability of the intron enhancer to stimulate binding of CstF via UV cross-linking and immunoprecipitation of cross-linked proteins (Fig. 4). A CT/CGRP exon 4 precursor RNA containing the wild-type intron enhancer demonstrated at least twofold greater UV cross-linking to the $64-\mathrm{kD}$ subunit of CstF than did precursor RNA containing an enhancer mutated for its internal 5' splice site sequence.

CstF normally binds to sequences near the cleavage site, and binding requires standard polyadenylation signals. Two control experiments indicated that CstF crosslinking to the exon 4 poly(A) site was similar to CstF cross-linking to other poly(A) sites. Cross-linking of CstF was dependent on the AAUAAA within the exon 4 poly(A) site (data not shown). In addition, no cross-linking of CstF could be observed to an RNA containing just the enhancer sequence (data not shown). These results suggest both that the enhancer affects polyadenylation cleavage by affecting the binding of CstF to the exon 4 poly(A) site and that the $5^{\prime}$ splice site sequence within the enhancer is required for maximal enhancement of $64-\mathrm{kD}$ binding.

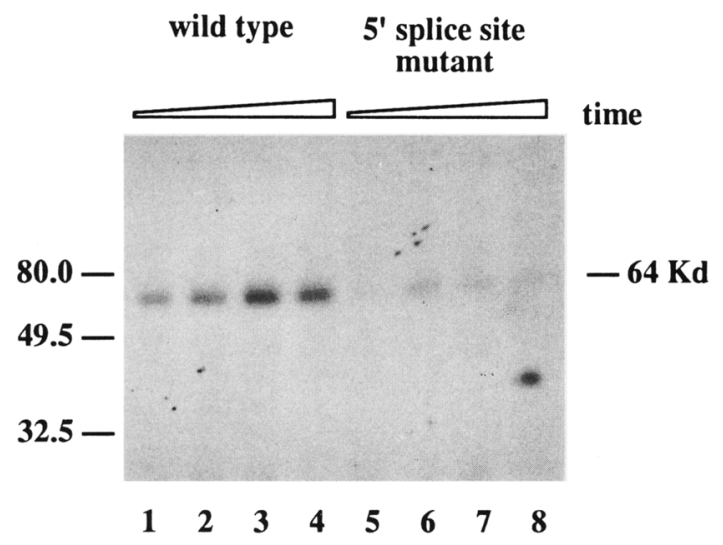

Figure 4. The CT/CGRP intron enhancer stimulates UV cross-linking of the $64-\mathrm{kD}$ polyadenylation factor to precursor RNAs containing the exon 4 poly(A) site. A standard polyadenylation reaction was sampled for UV cross-linking of the 64$\mathrm{kD}$ subunit of CstF to precursor RNA at 2, 5, 8, and 10 min. Cross-linked proteins were immunoprecipitated with an anti64-kD antibody (courtesy of C. MacDonald, Texas Tech University, Lubbock). Polyadenylation substrates included RNAs containing the CT/CGRP exon 4 poly(A) site with either the wild-type enhancer (left) or the mutant enhancer in which the pseudo-5' splice site within the enhancer had been altered from GUAAG to CAUAG (right).
Activation by the intron enhancer requires U1 RNA

A requirement for a pseudo- $5^{\prime}$ splice site within the enhancer suggested that U1 snRNPs play a role in enhancer activity via their ability to bind to $5^{\prime}$ splice sites. To test this hypothesis, in vitro polyadenylation cleavage assays were performed under conditions in which U1 RNA hybridization to $5^{\prime}$ splice sites was inhibited. To inhibit hybridization, polyadenylation extract was pretreated with an antisense 2 '-O-methyl ribo-oligonucleotide complementary to the $5^{\prime}$ terminal sequence of $U^{\prime}$ RNA. Inhibition of hybridization of Ul RNA to a 5' splice site following the antisense treatment was confirmed by analysis of standard in vitro splicing reactions (data not shown). Pretreatment of extract used for in vitro polyadenylation with the anti-U1 oligonucleotide inhibited polyadenylation cleavage of precursor RNAs containing the CT/CGRP exon 4 poly(A) site and the wild-type intron enhancer (Fig. 5A). In contrast, oligonucleotides directed against U1 RNA had no effect on polyadenylation cleavage of a control polyadenylation precursor RNA (the SV40 late poly(A) site) lacking any known distant downstream enhancer element. Control oligonucleotides had no effect on polyadenylation cleavage of RNAs containing either the exon 4 poly(A) site or the SV40 poly(A) site.

Especially definitive was an experiment using precursor RNAs containing the APRT poly(A) site to which the intron enhancer had been added. Polyadenylation cleavage of RNAs containing the native APRT site was not inhibited by hybridization of U1 RNA to a complementary ribo-oligonucleotide (Fig. 5B). In contrast, inactivation of U1 RNA inhibited polyadenylation cleavage of a precursor RNA containing the APRT site to which the intron enhancer had been added, and returned the observed level of polyadenylation cleavage to that of RNAs containing the APRT site without the added element. These results provide additional support for an interaction between U1 snRNPs and the $5^{\prime}$ splice site sequence within the intron enhancer, as well as a role for this complex in enhancer-mediated activation of polyadenylation cleavage.

\section{Protection of the enhancer-located 5' splice site requires U1 RNA}

The observation that U1 RNA is required for enhancermediated polyadenylation suggested that U1 RNA actually binds to the enhancer-located $5^{\prime}$ splice site sequence. To investigate this possibility, we performed protection experiments to see whether U1 RNA was required for binding of factors to the enhancer-located $5^{\prime}$ splice site (Fig. 6). For this experiment, extract was pretreated with an oligonucleotide complementary to nucleotides 1-14 of U1 RNA and RNase $\mathrm{H}$. This treatment effectively removed the $5^{\prime}$ sequences of U1 RNA, thereby prohibiting hybridization of UI RNA to $5^{\prime}$ splice site sequences. Binding of factors to the $5^{\prime}$ splice site within the enhancer was monitored by addition of an oligonucleotide complementary to the enhancer-located $5^{\prime}$ splice site at various times of the reaction (Eperon et 
A

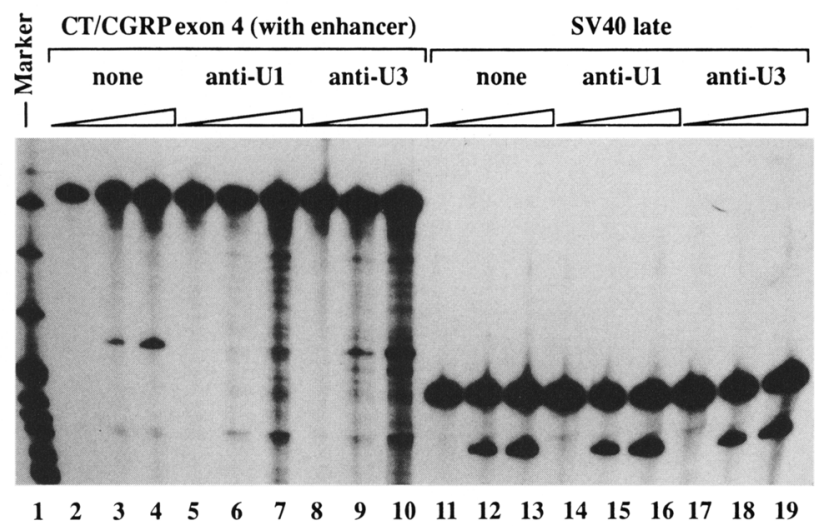

B

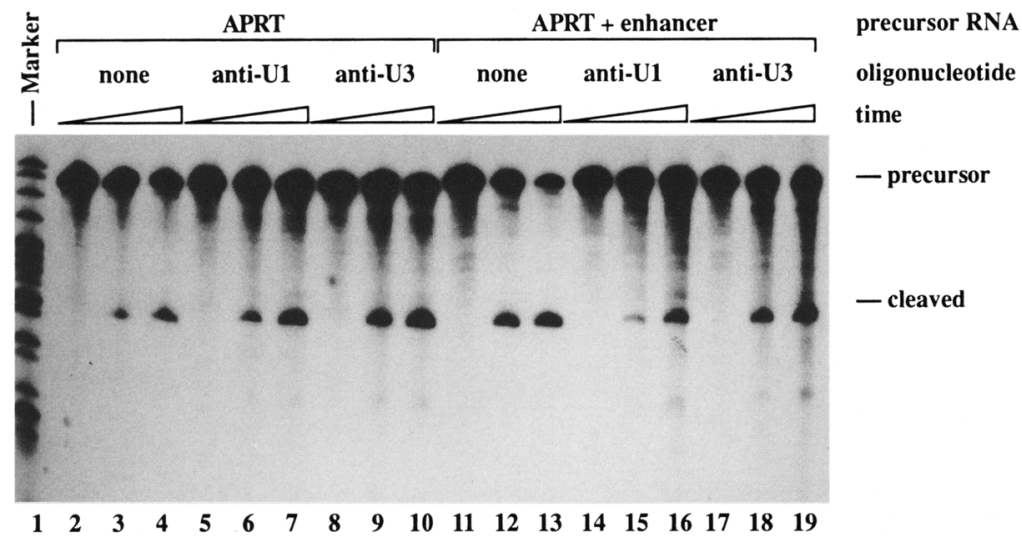

time precursor RNA

oligonucleotide

- CT/CGRP precursor

- CT/CGRP cleaved

- SV40 precursor

- SV40 cleaved

al. 1994; Zuo and Manley 1994; Jamison et al. 1995). To test for the binding of ASF/SF2 to the intron enhancer, we assayed the ability of ASF/SF2 to be UV cross-linked to both mutant and wild-type precursor RNAs. Wildtype RNA containing the exon 4 poly(A) site and the intron enhancer was cross-linked to ASF/SF2 as determined by immunoprecipitation of cross-linked proteins with an ASF/SF2-specific antibody (Fig. 7A). Cross-linking of ASF/SF2 was greatly reduced when a precursor RNA was used that contained the exon 4 poly(A) site and the intron enhancer mutated for its internal 5' splice site sequence.

An RNA containing only the intron enhancer sequence and no polyadenylation consensus sequences could also be UV cross-linked to ASF/SF2 (Fig. 7B). Just as with the intact exon 4 poly(A) site containing the intron enhancer, maximal cross-linking was dependent on the pseudo-5' splice site within the enhancer. These results suggest that the enhancer $5^{\prime}$ splice site sequence binds an $S / R$ protein.

\section{Additional sequence elements within the enhancer are important for in vitro polyadenylation cleavage}

The $5^{\prime}$ splice site of the intron enhancer is preceded by a polypyrimidine tract. Replacement of pyrimidines within the tract by purines depresses in vivo recognition of normally associated with $5^{\prime}$ splice sites. One such factor is the S/R protein ASF/SF2 (Eperon et al. 1993; Kohtz et 


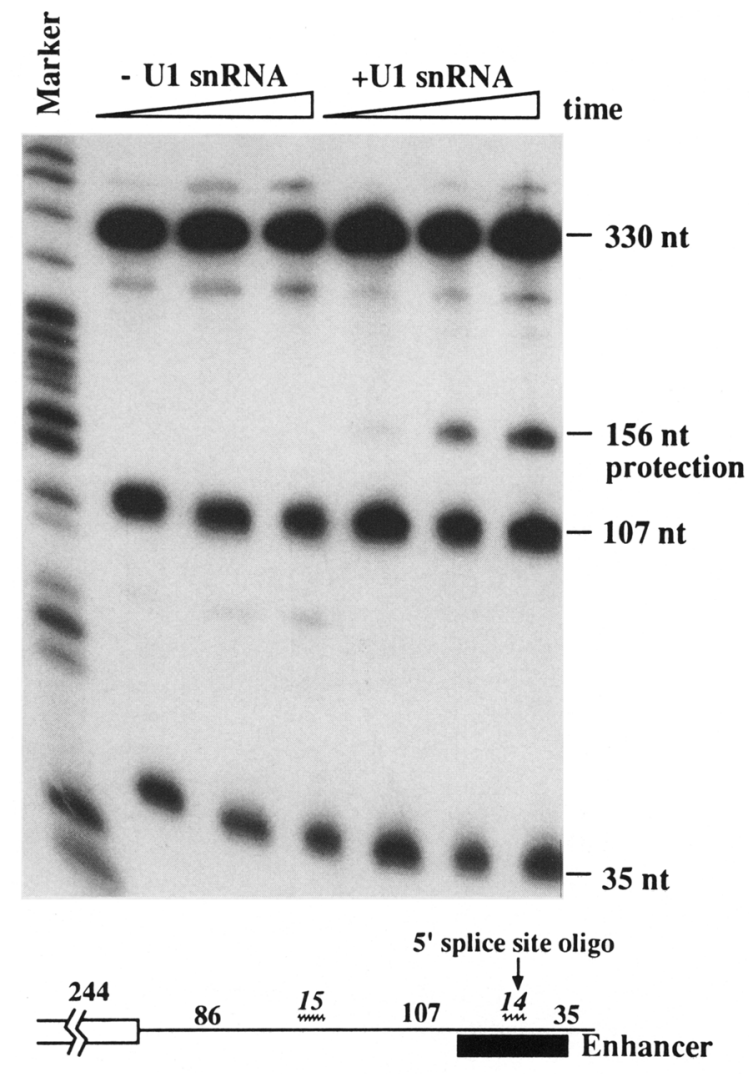

Cleavage products of $\mathrm{RNase} \mathrm{H}$ digestion:

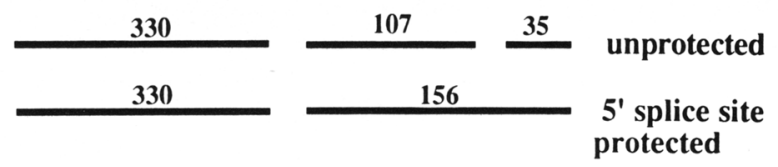

Figure 6. Binding of factors to the enhancer-located 5' splice site requires U1 RNA. Binding of factors to the enhancer-located $5^{\prime}$ splice site was monitored using a protection assay in which oligonucleotides complementary to the enhancer-located 5 ' splice site were used to direct precursor RNA cleavage in the presence of RNase $\mathrm{H}$. Wild-type precursor RNA containing the natural CT/CGRP exon 4 poly(A) site with the intron enhancer was incubated in a standard polyadenylation reaction $1+\mathrm{Ul}$ RNA, right) or in a reaction pre-treated with an oligonucleotide directed against nucleotides 1-14 of U1 RNA and RNase H/-Ul RNA, left). Treatment removed the terminal 14 nucleotides of U1 RNA, thereby inhibiting binding to $5^{\prime}$ splice sites. After incubation at $30^{\circ} \mathrm{C}$ for 0,5 , or $10 \mathrm{~min}$., two oligonucleotides complementary to two regions of the precursor RNA starting 86 or 208 nucleotides downstream of the poly(A) cleavage site (oligonucleotides indicated by wavy lines) were added to permit RNase $\mathrm{H}$ digestion of precursor RNA. RNAs were prepared and analyzed after incubation for $15 \mathrm{~min}$ following oligonucleotide addition. Expected cleavage products are diagrammed below the gel. The three major cleavage products of precursor RNA / 330 , 107 , and $35 \mathrm{nt}$ ) and the protection band resulting from binding of nuclear factors to the 14 nucleotide region of the enhancer containing the $5^{\prime}$ splice site (156 nt) are indicated.

CT/CGRP exon 4 (Lou et al. 1995), suggesting that the polypyrimidine tract of the enhancer might play a role in polyadenylation of exon 4 . To test this possibility, the effect of mutation of the polypyrimidine tract on in vitro polyadenylation cleavage was examined. The same mutation that depressed in vivo inclusion of exon 4 (Lou et al. 1995) was assayed in vitro. As shown in Figure 8A, mutation of the polypyrimidine sequence reduced polyadenylation cleavage of RNAs containing the exon 4 poly(A) site to $33 \%$ of the level for wild-type precursor RNA, suggesting that the pyrimidine tract plays a role in enhancer-mediated activation of polyadenylation cleavage. In contrast, mutation of a second pyrimidine tract within the enhancer downstream of the $5^{\prime}$ splice site had no effect on in vitro polyadenylation cleavage (data not shown). Thus, the important sequences in the intron enhancer revealed by our mutations are the pyrimidine tract and the adjacent pseudo-5' splice site.

\section{The pyrimidine tract of the enhancer binds PTB}

The polypyrimidine tract of the enhancer is immediately followed by a CAG sequence, an arrangement character-
A

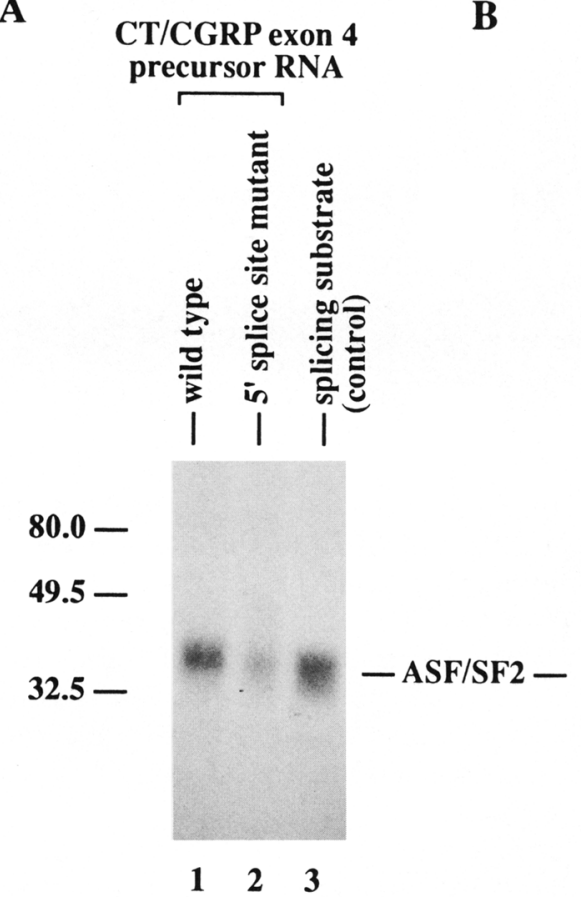

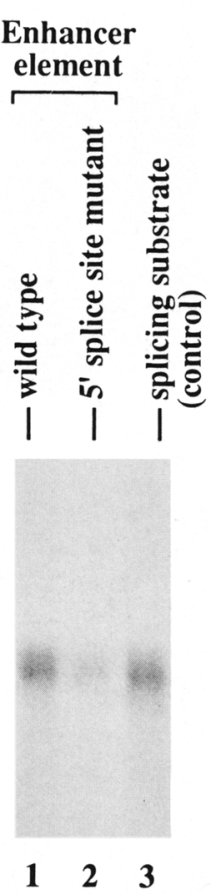

Figure 7. The intron enhancer binds ASF/SF2. Polyadenylation precursor RNAs were examined by UV cross-linking and immunoprecipitation of cross-linked proteins with a monoclonal antibody specific for ASF/SF2 (courtesy of A. Krainer, Cold Spring Harbor Laboratory, NY). Polyadenylation reactions were subjected to UV cross-linking after $5 \mathrm{~min}$ of incubation. (A) UV cross-linking analysis of RNAs containing the CT/CGRP exon 4 poly(A) site with either a wild-type enhancer (denoted wildtype) or an enhancer in which the $5^{\prime}$ splice site had been $\mathrm{mu}$ tated from GUAAG to CAUAG (denoted 5 ' splice site mutant). A cross-linking reaction using a standard adenovirus-based splicing precursor RNA (splicing substrate) was included as a control for ASF/SF2 binding to a 5 ' splice site. (B) UV crosslinking analysis of 182 nucleotide RNAs containing just the intron enhancer, wild-type or mutant, were incubated under polyadenylation conditions followed by UV cross-linking and immunoprecipitation with antibodies specific for ASF/SF2. 
A

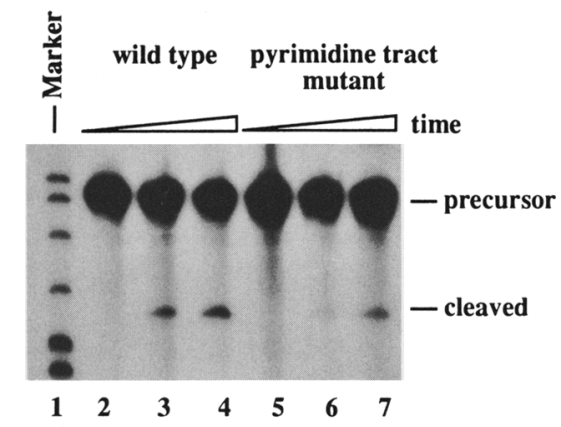

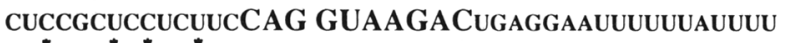
$\begin{array}{llllll}\mathbf{A} & & \mathbf{G} & \mathbf{A} & \mathbf{G}\end{array}$

B

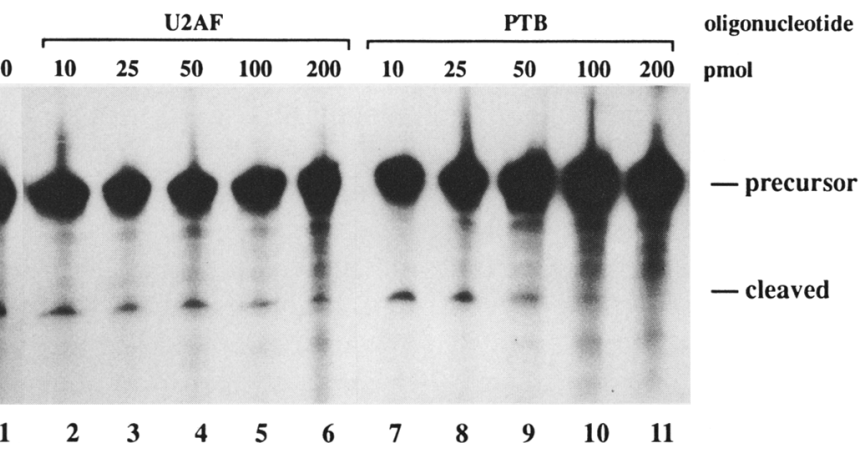

C

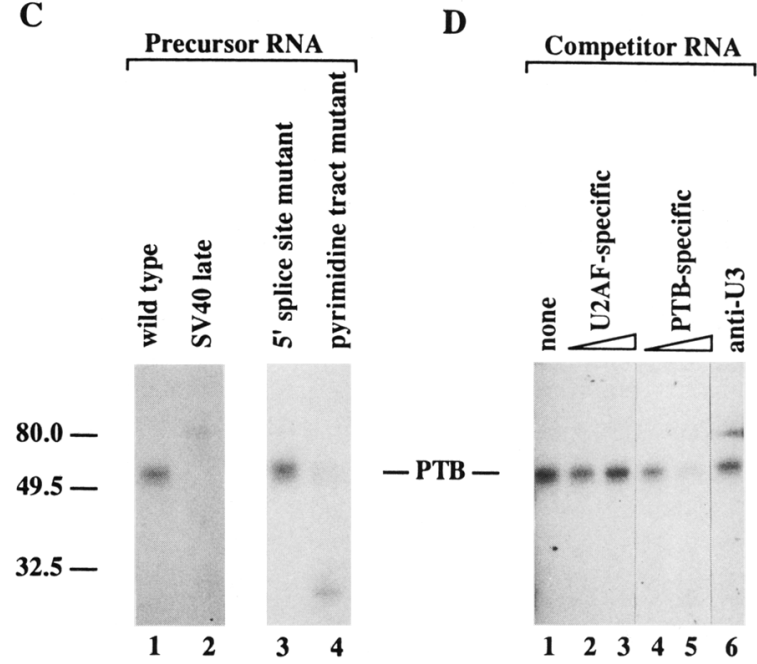

Figure 8. The pyrimidine tract of the intron enhancer is required for maximal polyadenylation cleavage and binds PTB. (A) Comparison of polyadenylation cleavage activity of precursor RNAs containing the wild-type enhancer or the enhancer with a mutated pyrimidine tract. The upstream pyrimidine tract was mutated via the replacement of four pyrimidines with purines as indicated. Polyadenylation precursor RNAs containing an enhancer bearing the mutant pyrimidine tract were assayed for polyadenylation cleavage in comparison to RNAs containing the wild-type pyrimidine tract. Time points were taken at 0,20 , and $40 \mathrm{~min}$. $(B)$ Effect of competitor RNAs specific for U2AF or PTB on enhancer-mediated polyadenylation cleavage. Polyadenylation cleavage assays with RNAs containing the CT/CGRP exon 4 poly $(\mathrm{A})$ site including the wild-type enhancer were performed in the presence of one of two competitor RNAs (5'-UUUUCCCUUUUUUUUC- $3^{\prime}$, containing a sequence among those selected by U2AF via reiterative selection, left; or 5'-GCCUGCUGCUCCUCUUCUGUC-3', containing a sequence among those selected by PTB via reiterative selection, right $)$. Utilized amounts of oligonucleotides are shown above each lane. Experiments utilized 5-10 fmoles of precursor RNA per assay. All samples were taken at $40 \mathrm{~min}$. (C) Comparison of PTB that UV cross-links to precursor RNAs containing wild-type or mutant enhancers. Polyadenylation reactions using the following RNAs were subjected to UV cross-linking after 5 min of incubation: CT poly(A) site with the wild-type intron enhancer (lane 1), SV40 late poly(A) site (lane 2), CT poly(A) site with the enhancer mutated for its $5^{\prime}$ splice site sequence (lane 3), or CT poly(A) site with the enhancer mutated for its pyrimidine tract sequence (lane 4). Cross-linked proteins were immunoprecipitated with antibodies specific for PTB (gift of D. Helfman, Cold Spring Harbor Laboratory, NY). (D) Effect of U2AF or PTB-specific oligonucleotides on binding of PTB. UV cross-linking of PTB to precursor RNAs containing the intron enhancer was competed by U2AF-specific (lanes 2, 3) or PTB-specific RNA oligonucleotides (lanes 4, 5) (0.3, 3.0 pmoles). A non-specific RNA oligonucleotide complementary to U3 RNA was included as a control (lane 6). The conditions were those described in $A$ except that the reaction was halted at $5 \mathrm{~min}$ for UV cross-linking.

istic of $3^{\prime}$ splice sites. The polypyrimidine tracts of $3^{\prime}$ splice sites are known to bind several proteins, notably $\mathrm{U} 2 \mathrm{AF}$ and PTB. Recently, preferred RNA-binding sites for U2AF and PTB have been determined by reiterative selection techniques (Singh et al. 1995). Both bind polypyrimidine sequences but have different preferred sequences, making it possible to assay for involvement of one or both of these proteins via ribo-oligonucleotide competition experiments.

To perform these experiments, increasing concentrations of ribo-oligonucleotide competitor RNAs containing the U2AF-selected sequence or the PTB-selected sequence (oligonucleotide sequences in Figure $8 \mathrm{~B}$ ) were preincubated with extract prior to the addition of in vitro polyadenylation cleavage substrates. For this experiment, we used the CT/CGRP exon 4 poly(A) site containing the intron enhancer. Oligonucleotides containing the U2AF-selected sequence had no effect on the ef- ficiency of polyadenylation cleavage, suggesting that U2AF plays no role in enhancer-mediated activation of polyadenylation cleavage. In contrast, oligonucleotides containing the PTB-selected sequence strongly inactivated polyadenylation cleavage, implicating a role for PTB in enhancer-mediated activation of polyadenylation cleavage.

As controls for this experiment, we asked if the U2AFspecific and PTB-specific oligonucleotides had an effect on the efficiency of polyadenylation cleavage of precursor RNAs containing other poly|A| sites lacking the enhancer. Neither oligonucleotide affected the efficiency of polyadenylation cleavage of RNAs containing the SV40 late poly(A) site (data not shown). In a separate experiment, neither oligonucleotide affected the ability of the 64-kD subunit of CstF to be UV cross-linked to RNAs containing a poly(A) site lacking the enhancer (data not shown). 
Lou et al.

To confirm that PTB binds to the intron enhancer, UV cross-linking assays were performed with polyadenylation precursor RNAs containing the wild-type or mutated enhancer sequence (Fig. 8C). Immunoprecipitation with antibodies specific for PTB indicated that PTB bound to precursor RNAs containing either the wildtype intron enhancer or the intron enhancer mutated for the pseudo 5' splice site. In contrast, little cross-linking was observed to a precursor RNA in which the intron enhancer pyrimidine tract had been mutated. This result suggests that PTB binds to the pyrimidine tract within the enhancer. PTB cannot normally be UV cross-linked to precursor RNAs containing poly(A) sites. As shown in Figure 8C, PTB was not cross-linked to a precursor RNA containing the SV40 late poly(A) site. Thus, the intron enhancer binds a protein not normally associated with poly(A) sites.

To correlate the results from cross-linking of PTB to the results from oligonucleotide inhibition of cleavage, the effect of addition of competitor oligonucleotides representing preferred binding sites for PTB or U2AF on cross-linking of PTB was assayed (Fig. 8D). Cross-linking of PTB to the wild-type exon 4 precursor RNA was inhibited by a PTB-specific competitor, but not by nonspecific oligonucleotides. The same concentration of an oligonucleotide containing the preferred U2AF binding site did not compete UV cross-linking of PTB. The ability of the same oligonucleotide to inhibit PTB cross-linking and polyadenylation cleavage suggests that PTB both binds to the enhancer and participates in its activity.

U2 snRNPs recognize and bind to 3 ' splice sites via an interaction with U2AF (Zamore and Green 1989, 1991). The previous experiments suggested that U2AF does not bind to the portion of the intron enhancer that resembled a 3 ' splice site. This failure suggested that U2 snRNPs would not be required for enhancer-mediated polyadenylation cleavage of the exon $4 \operatorname{poly}(\mathrm{A})$ site. To test this hypothesis, polyadenylation cleavage reactions were performed using extract in which U2 RNA was cleaved by the action of RNase $\mathrm{H}$ in the presence of oligonucleotides complementary to U2 sequences. Under conditions in which U2 RNA was completely cleaved and the splicing of standard splicing templates was inhibited by at least $95 \%$, there was no diminution of polyadenylation cleavage of precursor RNAs containing the exon 4 poly(A) site with the wild-type enhancer (data not shown). Therefore, U2 snRNA and presumably U2 snRNPs do not participate in enhancer-mediated polyadenylation. These results add further support to the conclusion that U2AF does not bind to the enhancer polypyrimidine tract.

\section{Discussion}

Regulation of alternative processing of CT/CGRP involves the differential recognition of a $3^{\prime}$-terminal exon, exon 4 . Exon 4 has both a weak $3^{\prime}$ splice site and a weak poly(A) site as determined both by sequence comparison and in vitro activity. In previous studies we identified an intron enhancer sequence located downstream of the exon 4 poly $(\mathrm{A})$ site. This sequence is required for the exon 4 inclusion in HeLa cells (Lou et al, 1995). Here we demonstrate that recognition of the exon 4 poly(A) site is stimulated by the presence of this intron enhancer sequence in both the human and mouse CT/CGRP genes. Mutations in the intron enhancer inhibited both in vivo recognition of exon 4 and in vitro polyadenylation cleavage of precursor RNAs containing the exon 4 poly(A) site. These results suggest that the CT/CGRP intron enhancer facilitates exon 4 recognition via an effect on polyadenylation cleavage at the exon 4 poly(A) site.

The CT/CGRP intronic sequence element defined here as an intron enhancer is distinct from the downstream sequences normally associated with poly(A) sites (for review, see in Wahle 1995). Positioned 168 nucleotides downstream of the polyadenylation cleavage site, it is located farther from the poly|A) site than other known polyadenylation regulators that are typically within 30-50 nucleotides of the AAUAAA. In vivo, the enhancer facilitates exon 4 recognition when placed 544 nucleotides downstream of exon 4 (Lou et al. 1995). To our knowledge, this is the first report of an intron enhancer located several hundred nucleotides from a polyadenylation cleavage site that functions to activate polyadenylation cleavage. Befitting the term enhancer, the CT/CGRP intronic element functions to activate heterologous poly(A) sites when placed downstream of those sites. Both the ability to function at a distance and the capacity to activate heterologous poly(A) sites suggest that the intron enhancer functions via protein-protein interactions to facilitate polyadenylation cleavage.

The CT/CGRP enhancer has an unusual structure in that it contains sequence elements normally associated with functional splice sites. A pyrimidine tract, CAG, and $5^{\prime}$ splice site sequence reside at the heart of the enhancer. Mutation of either the $5^{\prime}$ splice site or the pyrimidine tract inhibited in vivo exon 4 recognition and in vitro exon 4 polyadenylation cleavage. To our knowledge this is the first enhancer reported to contain a $5^{\prime}$ splice site. Consistent with the sequence being recognized as a splice site during enhancement of polyadenylation, the sequence bound both U1 snRNPs and ASF/SF2. Enhancement of polyadenylation cleavage and protection of the enhancer-located $5^{\prime}$ splice site required the $5^{\prime}$ terminal sequence of U1 RNA, suggesting that Ul snRNPs both recognize the enhancer and participate in polyadenylation.

A positive role for U1 snRNPs in constitutive polyadenylation has been suggested by the ability of the U1 snRNP A polypeptide to bind to activator sequences located upstream of the SV40 poly(A) site (Lutz and Alwine 1994). No evidence for a role for U1 RNA in this activation has been documented in that in vitro polyadenylation of precursor RNAs containing the SV40 poly(A) site does not require the $5^{\prime}$-terminal sequences of U1 RNA. These results suggest that activation of polyadenylation by the U1 snRNP A protein does not require hybridization of U1 RNA to sequences resembling $5^{\prime}$ splice sites located near a poly(A) site (Wassarman and Steitz 1993). Activation of polyadenylation cleavage by the $\mathrm{CT} /$ 
CGRP intron enhancer, however, did require the 5 '-terminal sequences of U1 RNA, suggesting both that U1 RNA hybridizes to the 5 ' splice site within the intron enhancer and that U1 snRNPs so bound can facilitate polyadenylation of an upstream distal poly(A) site.

The CT/CGRP enhancer contains a pyrimidine tract in addition to a $5^{\prime}$ splice site. This pyrimidine tract is located upstream of the $5^{\prime}$ splice site, and a CAG sequence separates the pyrimidine tract from the $5^{\prime}$ splice site. This arrangement of sequences is similar to that seen across an internal exon except that in the enhancer there is no sequence between the CAG of the $3^{\prime}$ splice site-like sequence and the GUAAG sequence of the $5^{\prime}$ splice site. Thus, the arrangement of sequences is not optimal for splicing. Normally, pyrimidine tracts in vertebrate 3' splice sites bind U2AF and U2AF binding is important for exon recognition (Zamore and Green 1989; 1991; Hoffmann and Grabowski 1992; Wang et al. 1995). Because the splice sites within the CT/CGRP enhancer are not used to direct active splicing, it would be predicted that the pyrimidine tract of the enhancer does not productively interact with U2AF or U2 snRNPs. Competition experiments using ribo-oligonucleotides specific for a polypyrimidine tract selected by U2AF in reiterative selection indicated that U2AF binding was not required for enhancer function. Similarly, U2 snRNPs did not appear to be involved in enhancement.

Enhancer-mediated polyadenylation was dependent on PTB, a factor that binds polypyrimidine tracts but whose binding has been associated with inhibition of exon recognition rather than enhancement of exon recognition (Mulligan et al. 1992; Lin and Patton 1995; Singh et al. 1995). PTB was efficiently UV cross-linked to the CT/ CGRP poly(A) site including the wild-type enhancer, but not when the pyrimidine tract within the enhancer was mutated. Oligonucleotides representing preferred PTB binding sites inhibited enhancer-mediated polyadenylation cleavage and cross-linking of PTB. The pyrimidine tract of the enhancer, CUCCGCUCCUCUUC, resembles the PTB-selected sequence used in these studies as a competitor, CUGCUCCUCUUCU, in that it is a mixture of U's and C's interrupted with a single G. Therefore, the binding of PTB to these sequences is perhaps not startling. Recently, PTB has been subjected to reiterative selection using even greater rounds of selection than in the study by Singh et al. (1995). In these experiments a more definitive sequence for PTB binding emerged in which the core sequence UCUU was present in all selected oligonucleotides (J.G. Patton, pers. comm.). Both the human and murine CT/CGRP enhancer contain a UCUU within the pyrimidine tract, further supporting a role for PTB in enhancer-mediated polyadenylation.

From our observations we suggest a model in which the intron-located $5^{\prime}$ splice site of the CT/CGRP enhancer functions to bind Ul snRNPs in a fashion such that they can facilitate polyadenylation. Binding of the pyrimidine tract of the enhancer to PTB would, by analogy to other systems of differential splicing in which PTB binding has been correlated with inhibition of exon recognition, prevent productive recognition of the enhancer-located $5^{\prime}$ splice site for splicing. Thus, we suggest that PTB binding to the enhancer functions to silence the enhancer for splicing and activate it for polyadenylation.

Recognition of CT/CGRP exon 4 occurs in a variety of tissues when the CT/CGRP gene is introduced into transgenic mice, suggesting that the intron enhancer functions in many tissues and is recognized by widely distributed processing factors. The three factors that we have implicated in enhancer recognition, Ul snRNPs, PTB, and ASF/SF2 have such a broad distribution although the concentrations of the latter two vary from tissue to tissue (Patton et al. 1991; Zahler, and Roth 1993). In a few tissues, especially neuronal cells, CT/ CGRP exon 4 is not recognized. The relationship of the intron enhancer to loss of recognition of CT/CGRP exon 4 in neuronal cells is unclear. The enhancer could be the target of factors that inhibit exon 4 recognition. The mutations characterized in this study that inhibit in vivo recognition of exon 4 and in vitro polyadenylation of the exon 4 poly(A) site, however, do not cause recognition of exon 4 in cell lines that normally skip the exon (Lou et al. 1995), suggesting that the sequences within the enhancer that bind positively acting factors are not those that also bind negatively acting factors in neuronal cells. Because all of our mutants alter sequences that direct positive events for exon 4 inclusion, however, it is not clear that such mutations should have a phenotype in neuronal cells. It remains a possibility that neuronal cells are lacking or have reduced quantities of factors that normally recognize some element of the enhancer sequence, such as ASF/SF2 or PTB. In this scenario, recognition of the intron enhancer would be the definitive event in the regulation of CT/CGRP alternative splicing.

\section{Materials and methods}

\section{Plasmids}

The wild-type CT clone used for in vivo expression was created by fusing the full-length human CT genomic sequence (Cote et al. 1990) to the SacI-EcoRI enhancer fragment of the RSV promoter. A $5^{\prime}$ splice site mutation (GTA to CAT at positions +1 to +3 ) was introduced into the intron enhancer region by PCRdirected mutagenesis.

To make templates for in vitro transcription, the following poly (A) sites were subcloned in pGEM3Zf $|+|$ vector (Promega): NsiI-Ncol fragment of human CT exon $4, N s i I-X b a I$ fragment of mouse CT exon 4, and PvuII-PstI fragment of hamster APRT exon 5. The human CT exon 6 poly(A) site was PCR amplified and then subcloned because of the lack of convenient restriction sites. The lengths of exon sequence and the sequence downstream from the poly(A) sites are shown in Figures 2 and 3. The $\mathrm{CT}$ exon 4 poly $(\mathrm{A})$ sites with mutated intron enhancers were subcloned from the in vivo expression constructs (Lou et al. 1995). The 127-nucleotide wild-type and mutated CT intron enhancer sequence was fused downstream of the APRT and CT exon 6 poly(A) sites to create the enhanced poly(A) sites. All of the clones were confirmed by sequencing.

HeLa cell transfection and RT/PCR analysis

HeLa cells transfections and RNA isolation were described pre- 
viously (Lou et al. 1994). Splicing phenotypes were assayed by RT/PCR. Fifteen-cycle PCR amplification with ${ }^{32} \mathrm{P}$-labeled primer was performed to determine the relative abundance of individual RNA species. Primers specific for both exons 4 and 5 were used during amplification to permit simultaneous visualization of RNAs resulting from inclusion and skipping of exon 4. Details of the utilized RT/PCR procedure, including primer sequence, have been described previously (Lou et al. 1995).

\section{In vitro polyadenylation}

RNA transcripts for in vitro polyadenylation cleavage and UV cross-linking reactions were synthesized by SP6 RNA polymerase (GIBCO-BRL) in presence of $50 \mu \mathrm{Ci}$ of [ ${ }^{32} \mathrm{P}$ ]UTP (DuPont). RNAs were gel-purified, eluted, and ethanol precipitated. Polyadenylation cleavage reactions were carried out in a volume of $50 \mu \mathrm{l}$ containing $40 \%$ (vol/vol) HeLa cell nuclear extract, 0.1 mM ATP, $20 \mathrm{~mm}$ creatine phosphate, $1.0 \mathrm{~mm} 3^{\prime}$-dATP, $0.6 \mathrm{~mm}$ $\mathrm{MgCl}_{2}, 1.5 \%$ PEG, $0.15 \mathrm{mM}$ DTT, and $5 \times 10^{4} \mathrm{cpm}$ of ${ }^{32} \mathrm{P}$-labeled RNA. 3'-dATP was included to terminate the poly(A) addition reaction. The reactions were incubated at $30^{\circ} \mathrm{C}$ and the aliquots were taken at 20 and $40 \mathrm{~min}$ followed by phenol/chloroform (1:1) extraction and ethanol precipitation. The RNAs were analyzed on $5 \%$ polyacrylamide gels containing $8.3 \mathrm{M}$ urea.

To inhibit U1 snRNA base pairing with $5^{\prime}$ splice site sequences, polyadenylation reactions were preincubated with 1 nmol of a 2'-O-methyl ribo-oligonucleotide (Oligos Etc.) complimentary to the first 14 nucleotides of U1 snRNA prior to the addition of substrate. A 2'-O-methyl ribo-oligonucleotide complementary to the first 12 nucleotides of U3 snRNA was used as a negative control. Cleavage of U1 RNA was accomplished by pretreatment of extract with an deoxyoligonucleotide complementary to nucleotides $1-14$ of U1 RNA and RNase $\mathrm{H}\{1.5 \mathrm{U}$, Promega). Pretreatment was long enough to affect degradation of remaining oligonucleotide. Competitor RNAs for the binding of U2AF and PTB were modeled after those identified using reiterative selection (Singh et al. 1995) Oligonucleotides specific for U2AF and PTB were 5 '-UUUUCCCUUUUUUUUC-3' (U2AF) and 5'-AGCCUGCUGCUCCUCUUCUGUC-3' (PTB). Oligonucleotides were incubated with polyadenylation reactions for $5 \mathrm{~min}$ on ice prior to the addition of precursor RNA.

Protection studies were performed via the addition of oligonucleotides complementary to a sequence located 82 nucleotides downstream of the exon 4 poly(A) site (GTC. CAGCTAGCCTAG) and to the enhancer-located $5^{\prime}$ splice site located 208 nucleotides downstream of the exon 4 poly(A) site (CAGTCTTACCTGGA). The second oligonculeotide was included to cut the 500 nucleotide precursor RNA into three fragments to better distingiush the protected bands from the unprotected. Incubation was continued for an additional $15 \mathrm{~min}$ following addition of the oligonucleotides to cause precursor RNA cleavage.

\section{UV cross-linking}

In vitro cleavage reactions were performed as indicated above except that $2 \times 10^{5} \mathrm{cpm}$ of substrates were used. Reactions were incubated at $30^{\circ} \mathrm{C}$ for $10 \mathrm{~min}$ or for the designated time, heparin was added to a final concentration of $2 \mu \mathrm{g} / \mu \mathrm{l}$, followed by UV irradiation at $4^{\circ} \mathrm{C}$ for $10 \mathrm{~min}$. Reactions were subsequently treated with $30 \mu \mathrm{g}$ of RNase A at $37^{\circ} \mathrm{C}$ for $30 \mathrm{~min}$. Cross-linked polypeptides were immunoprecipitated using monoclonal antibodies against the CstF $64-\mathrm{kD}$ subunit (gift from C. MacDonald), ASF/SF2 (gift from A. Krainer), or PTB (gift from D. Helfman). Immunoprecipitated proteins were separated on $10 \%$ SDS-polyacrylamide gels.

\section{Acknowledgments}

We thank Yun Yang for her assistance in DNA preparation and sequencing. We thank Clinton MacDonald, Adrian Krainer, and David Helfman for providing antibodies. We acknowledge the helpful advice of members in the Gagel and Berget laboratories. HeLa polyadenylation extract was prepared from cells grown by the Cell Culture Center in Minneapolis, MN. This work was supported by grants from The U.S. Public Health Servie (RO1DK38146 to RFG; and 2P30-CA16672 to M.D. Anderson Cancer Center) and the American Cancer Society (NP-695 to S.M.B.)

The publication costs of this article were defrayed in part by payment of page charges. This article must therefore be hereby marked "advertisement" in accordance with 18 USC section 1734 solely to indicate this fact.

\section{References}

Adema, G.J., R.A.L. Bovenberg, H.S. Jansz, and P.D. Baas. 1988. Unusual branch point selection involved in splicing of the alternatively processed calcitonin/CGRP-I pre-mRNA. $\mathrm{Nu}$ cleic Acids Res. 16: 9513-9526.

Adema, G.J., R.A.L. van Hulst, and P. D. Baas. 1990. Uridine branch acceptor is a cis-acting element involved in regulation of the alternative processing of calcitonin/CGRP-I premRNA. Nucleic Acids Res. 18: 5365-5372.

Amara, S.G., V. Jonas, and M.G. Rosenfeld. 1982. Alternative RNA processing in calcitonin gene expression generates mRNAs encoding different polypeptide products. Nature 298: 240-244.

Benett, M.M., and S.G. Amara. 1993. Molecular mechanisms of cell-specific and regulated expression of the calcitonin/ $\alpha$ CGRP and $\beta$-CGRP genes. Ann. N.Y. Acad. Sci. 257: 36-49.

Black, D.L. 1992. Activation of $c$-src neuron-specific splicing by an unusual RNA element in vivo and in vitro. Cell 69: 795807.

Boelens, W.C., E.J.R. Jansen, W.J van Venrooij, R. Stripecke, I.W. Mattaj, and S.I. Gunderson. 1993. The human Ul snRNPspecific U1A protein inhibits polyadenylation of its own premRNA. Cell 72: 881-892.

Broad, P.M., A.J. Symes, and R.K. Craig. 1989. Structure and methylation of the human calcitonin/ $\alpha$-CGRP gene. Nucl. Acids Res. 17: 6999-7011.

Cáceres, J.F., S. Stemm, D.M. Helfman, and A.R. Krainer. 1994. Regulation of alternative splicing in vivo by overexpression of antagonistic splicing factors. Science 265: 1706-1709.

Cote, G.J., R.V. Abrussese, C.J. Lips, and R.F. Gagel. 1990. Transfection of calcitonin gene regulatory elements into a cell culture model of the C cell. J. Bone Miner. Res. 5: 165171.

Cote, G.J., N. Nguyen, C.J.M. Lips, S.M. Berget, and R.F. Gagel. 1991. Validation of an in vitro RNA processing system for CT/CGRP precursor mRNA. Nucleic Acids Res. 19: 36013606.

Cote, G.J., D.T. Stolow, S. Peleg, S.M. Berget, and R.F. Gagel. 1992. Identification of exon sequences and an exon binding protein involved in alternative RNA splicing of calcitonin/ CGRP. Nucleic Acids Res. 20: 2361-2366.

Crenshaw, E.B., A.F. Russo, L.W. Swanson, and MG. Rosenfeld. 1987. Neuron-specific alternative RNA processing in transgenic mice expressing a metallothionein-calcitonin fusion gene. Cell 49: 389-398.

de Boer, J.G., E.A. Drobetsky, A.J. Grosovsky, M. Mazur, and B.W. Glickman. 1989. The Chinese hamster APRT gene as a mutational target. Its sequence and an analysis of direct and inverted repeats. Mutat. Res. 226: 239-244. 
Eperon, I.C., D.C. Ireland, R.A. Smith, A. Mayeda, and A.R. Krainer. 1993. Pathways for selection of $5^{\prime}$ splice sites by U1 snRNPs and SF2/ASF. EMBO $/$. 12: 3607-3617.

Ge, H. and J.L. Manley. 1990. A protein factor, ASF, controls cell-specific alternative splicing of SV40 early pre-mRNA in vitro. Cell 62: 25-34.

Hodges, D. and S.I. Bernstein. 1994. Genetic and biochemical analysis of alternative RNA splicing. Adv. Genet. 31: 207281.

Hoffmann, B.E. and P.J. Grabowski. 1992. Ul snRNP targets an essential splicing factor, $\mathrm{U}_{2} \mathrm{AF}^{65}$, to the $3^{\prime}$ splice site by a network of interactions spanning the exon. Genes \& Dev. 6: 2554-2568

Horabin, I., and P. Schedl. 1993. Sex-lethal autoregulation requires multiple cis-acting elements upstream and downstream of the male exon and appears to depend largely on controlling the use of the male exon 5' splice site. Mol. Cell. Biol. 13: 7734-7746.

Huh, G.S., and R. Hynes. 1993. Elements regulating an alternatively spliced exon of the rat fibronectin gene. Mol. Cell. Biol. 13: 5301-5314.

- 1994. Regulation of alternative pre-mRNA splicing by a novel repeated hexanucleotide element. Genes \& Dev. 8: $1561-1574$

Jamison, S.F, Z. Pasman, J. Wang, C. Will, R. Luhrmann, J.L. Manley, and M. Garcia-Blanco. 1995. U1 snRNP-ASF/SF2 interaction and 5 ' splice site recognition: characterization of required elements. Nucleic Acids Res. 23: 3260-3267.

Kohtz, J.D., S.F. Jamison, C.L. Will, P. Zuo, R. Lührmann, M.A. Garcia-Blanco, and J.L. Manley. 1994. Protein-protein interactions and $5^{\prime}$-splice site recognition in mammalian mRNA precursors. Nature 368: 119-124.

Krainer, A.R., G.C. Conway, and D. Kozak. 1990. The essential pre-mRNA splicing factor SF2 influences 5 ' splice site selection by activating proximal sites. Cell 62: 35-42.

Lin, C.-H. and J.G. Patton. 1995. Regulation of alternative $3^{\prime}$ splice site selection by constitutive splicing factors. RNA 1: 234-245.

Lou, H., G.J. Cote, and R.F. Gagel. 1994. The calcitonin exon and its flanking intronic sequences are sufficient for the regulation of human calcitonin/calcitonin gene-related peptide alternative splicing. Mol. Endocrinol. 8: 1618-1626.

Lou, H., Y. Yang, G.J. Cote, S.M. Berget, and R.F. Gagel. 1995. An intron splicing enhancer containing a $5^{\prime}$ splice site sequence in the human calcitonin/calcitonin gene-related peptide gene. Mol. Cell. Biol. 15: 7135-7142.

Lutz, C.S., and J.C. Alwine. 1994. Direct interaction of the U1 snRNP-A protein with the upstream efficiency element of the SV40 late polyadenylation signal. Genes \& Dev. 8: 57686.

MacDonald, C.C., J. Wilusz, and T. Shenk. 1994. The 64kilodalton subunit of the CstF polyadenylation factor binds to pre-mRNAs downstream of the cleavage site and influences cleavage site location. Mol. Cell. Biol. 14: 6647-6654.

Mayeda, A. and A.R. Krainer. 1992. Regulation of alternative pre-mRNA splicing by hnRNP Al and splicing factor SF2. Cell 68: 365-375.

Mulligan, G.J., W. Guo, S. Wormsley, and D.M. Helfman. 1992. Polypyrimidine tract binding protein interacts with sequences involved in alternative splicing of $\beta$-tropomyosin pre-mRNA. J. Biol. Chem. 267: 25480-25487.

Patton, J.G., S.A. Mayer, P. Tempst, and B. Nadal-Ginard. 1991. Characterization and molecular cloning of polypyrimidine tract-binding protein; a component of a complex necessary for pre-mRNA splicing. Genes \& Dev. 5: 1237-1251.
Siebel, C.W., L.D. Fresco, and D.C. Rio. 1992. The mechanism of somatic inhibition; Multiprotein complexes at an exon pseudo-5' splice site control U1 snRNP binding. Genes \& Dev. 6: 1386-1401.

Siebel, C.W., R. Kanaar, and D.C. Rio. 1994. Regulation of tissue-specific P-element pre-mRNA splicing requires the RNA-bindng protein PSI. Genes \& Dev. 8: 1713-1725.

Singh, R., J. Valcárcel, and M.R. Green. 1995. Distinct binding specificities and functions of higher eukaryotic polypyrimidine tract-binding proteins. Science 268: 1173-1176.

Takagaki, Y., J.L. Manley, C.C. MacDonald, J. Wilusz, and T. Shenk. 1990. A multisubunit factor, CstF, is required for polyadenylation of mammalian pre-mRNAs. Genes \& Dev. 4: 2112-2120.

van Oers, C.C.M., G.J. Adema, H. Zandberg, Y.C. Moen, and P.D. Baas. 1994a. Two different sequence elements within exon 4 are necessary for calcitonin-specific splicing of the human calcitonin/calcitonin gene-related peptide I premRNA. Mol. Cell. Biol. 14: 951-960.

van Oers, C.C.M., L. Bakker, and P.D. Baas. 1994b. The exon 4 poly(A) site of the human calcitonin/CGRP-I pre-mRNA is a weak site in vitro. Biochem. Biophys. Acta 1218: 55-63.

Wahle, E. 1995. 3'-Ends cleavage and polyadenylation of mRNA precursors. Biochem. Biophys. Acta 1261: 183-194.

Wang, Z., H.M. Hoffman, and P.J. Grabowski. 1995. Intrinsic U2AF binding is modulated by exon enhancer signal in parallel with changes in splicing activity. RNA 1: 21-35.

Wassarman, K.M., and J.A. Steitz. 1993. Association with terminal exons in pre-mRNAs: a new role for the U1 snRNP? Genes \& Dev. 7: 647-659.

Zahler, A.M., K.M. Neugebauer, W.S. Lane, and M.B. Roth. 1993. Distinct functions of SR proteins in alternative premessenger RNA splicing. Science 260: 219-222.

Zamore, P.D., and M.R. Green. 1989. Purification and biochemical characterization of U2 small nuclear ribonucleoprotein auxiliary factor. Proc. Natl. Acad. Sci. 86: 9243-9247.

. 1991. Biochemical characterization of U2 snRNP auxiliary factor; an essential pre-mRNA splicing factor with a novel intranuclear distribution. EMBO J. 10: 207-214.

Zhuang, Y., and A.M. Weiner. 1986. A compensatory base change in U1 snRNA suppresses a $5^{\prime}$ splice site mutation. Cell 46: 827-835.

Zuo, P. and J.L. Manley. 1994. The human splicing factor ASF/ SF2 can specifically recognize pre-mRNA $5^{\prime}$ splice sites. Proc. NatI. Acad. Sci. 91: 3363-3367. 


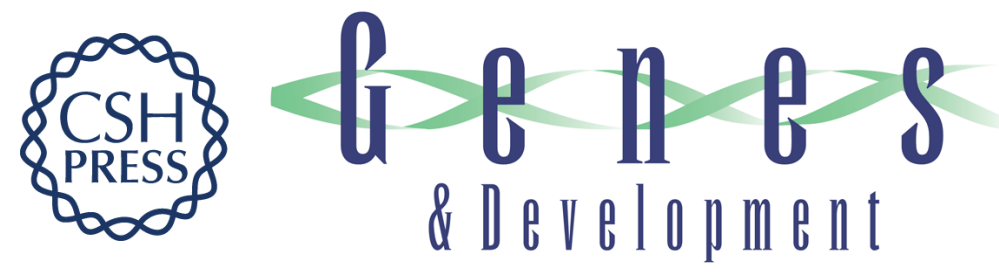

\section{An intron enhancer recognized by splicing factors activates polyadenylation.}

H Lou, R F Gagel and S M Berget

Genes Dev. 1996, 10:

Access the most recent version at doi:10.1101/gad.10.2.208

References This article cites 45 articles, 21 of which can be accessed free at:

http://genesdev.cshlp.org/content/10/2/208.full.html\#ref-list-1

License

Email Alerting

Service

Receive free email alerts when new articles cite this article - sign up in the box at the top right corner of the article or click here.

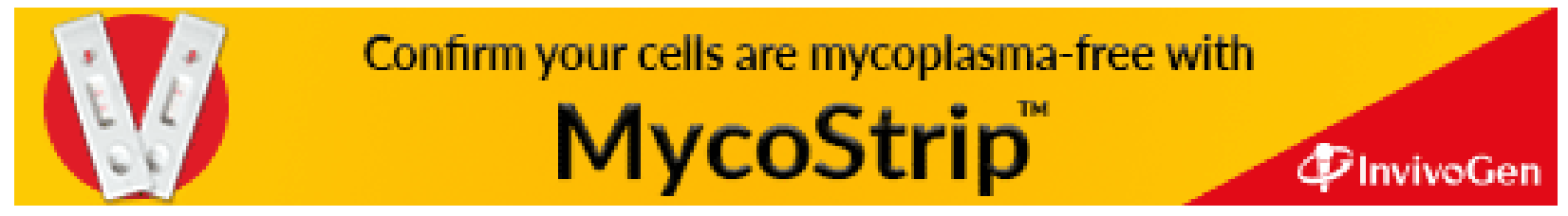

